\title{
Research
}

\section{Pre-hospital advanced life support resuscitation - a curriculum for pre-hospital education}

David Reid MHM(Hons), GradCertHSM, BSci(Paramedic Science) is Senior Lecturer and Director of Paramedicine'; Moira G Sim MBBS, PGDipDrugAlcAbStud, FRACGP, FAChAM, is Professor and Executive Dean ${ }^{1}$; Shelley Beatty DipTeach, BEd, PostgradDi(HlthProm), MPH, PhD is Associate Dean (Teaching and Learning) ${ }^{1}$; Hugh Grantham ASM, MBBS, FRACGP is Professor of Paramedics ${ }^{2}$,Senior Medical Practitioner ${ }^{3}$ and Senior Medical Officer ${ }^{4}$; Mike Gale BSc(Hons) Nursing, RN is National Course Coordinator ${ }^{5}$

\author{
Affiliations: \\ ${ }^{1}$ School of Medical and Health Sciences, Edith Cowan University, Perth, Western Australia \\ ${ }^{2}$ Curtin University, Perth, Western Australia \\ ${ }^{3}$ South Australian Ambulance Service, South Australia \\ ${ }^{4}$ Emergency Department, Flinders Medical Centre, Adelaide, South Australia \\ ${ }^{5}$ Australasian Resuscitation Council, Melbourne, Victoria
}

https://doi.org/10.33151/ajp.17.757

\section{Abstract}

\section{Introduction}

There is a broad evidence base to support advanced life support (ALS) education for healthcare professionals being structured, realistic and inclusive of a range of human factors. This paper outlines an ALS resuscitation education curriculum for providers working in the pre-hospital or resource-limited settings. The focus on pre-hospital ALS education is important because actions taken by pre-hospital healthcare professionals have a critical impact on the likelihood of patient survival.

\section{Methods}

The pre-hospital ALS curriculum developed in this research was derived from a survey and interviews with pre-hospital healthcare professionals and first responders, and input from resuscitation, medical and education experts. Following completion of the interviews an expert panel of resuscitation experts was consulted.

\section{Results}

Information collected indicated that a pre-hospital ALS course should follow current recommendations of the Australian Resuscitation Council and that the course should be delivered in a mixed mode comprising of online pre-reading and a quiz followed by one day of face-to-face teaching. Equipment should reflect that used in the pre-hospital environment and a pre-hospital ALS course should include scenarios relevant to the pre-hospital setting involving varying numbers of interprofessional healthcare teams as well as first responders and lay persons. Candidates should be assessed on a continual basis and certificates of participation be valid for 2 to 3 years.

\section{Conclusion}

To improve pre-hospital resuscitation education and maximise the likelihood of patient survival, ALS education for pre-hospital providers should follow Australian Resuscitation Council guidelines, include pre-course reading, case studies and practical simulation in situations that are likely to be encountered by pre-hospital professionals.

\section{Keywords:}

paramedic, education, resuscitation, pre-hospital, advanced life support training

Corresponding Author: David Reid, d.reid@ecu.edu.au 


\section{Background}

This paper proposes a curriculum for pre-hospital advanced life support (ALS) education. Previous research (1) found that the pre-hospital environment involves the unique challenges of unpredictable environment and resources, with limited space, suboptimal lighting and unpredictable bystander behaviour (2). Pre-hospital providers may also have to move and perform resuscitation procedures on a narrow stretcher and/or in a moving vehicle. In contrast, traditional training for managing cardiac arrests usually occurs in a hospital environment, with standardised equipment, sufficient space and lighting, fixed beds and a team of experienced staff. The optimum training for the management of cardiac arrests within the pre-hospital environment must differ from that within a healthcare facility. Lyon and Sinclair (2) argued that 'a specifically designed national pre-hospital ALS course would ensure the resuscitation standards of pre-hospital clinicians are maintained at the consistent and high level required'.

The International Liaison Committee for Resuscitation (ILCOR), European Resuscitation Council (ERC) and Australian Resuscitation Council (ARC) identify education as being one of the key attributes in improving outcomes from cardiac arrest (3-5). A literature review identified the important components in resuscitation education as: the mode of delivery; assessment; simulation and equipment; human factors; and workplace implementation. Resuscitation education was most effective when conducted with a face-to-face component $(1,6,7)$. Teaching in a similar environment to participants' workplaces, promotes congruence between the education and work environment $(3,8)$. Competency assessment was an essential component of ALS education $(5,9)$. Simulation was recognised as a safe way to teach and practise skills without endangering patients' lives (10-14). Human factors, or nontechnical skills have become increasingly recognised as critical components in ALS education $(9,15)$ with critical factors in ALS education being leadership, interprofessional teamwork and communication $(9,16-19)$.

The recognition that the pre-hospital environment presents unique challenges provides a compelling motivation to develop a specific evidence-based pre-hospital ALS program.

\section{Methods}

The proposed pre-hospital ALS curriculum was developed from data collected via an online survey and interviews with prehospital healthcare professionals and first responders, followed by input from an expert panel of ALS, medical and education experts, all with an interest in and exposure to pre-hospital resuscitation.

\section{Online survey}

An online survey, aligned with the Checklist for Reporting
Results of Internet E-Surveys (CHERRIES) recommendations for improving the quality of web surveys (20) collected information on the content pre-hospital professionals thought should be incorporated in a pre-hospital ALS training course. The survey was administered using Qualtrics $₫$ software between 1 March and 20 May 2018 with a convenience sample of 140 healthcare professionals including medical practitioners, nurses, paramedics and first responders, all with pre-hospital care experience.

Before data collection, face and content validity of the questionnaire items were assessed by administering the survey with six experienced pre-hospital healthcare providers who were also university paramedicine lecturers. The final survey contained quantitative and qualitative elements and had four sections including: survey introduction and informed consent; respondent demographics (two items); course preparation (six items); course length (two items); delivery method (four items); theoretical course elements (five items); human factors (two items); resuscitation skills (two items); simulations (scenarios) (four items); teamwork (one item); roles and responsibilities (four items); assessment (two items); standards and course governance (three items); and final comments and open-ended responses (three items).

\section{Interviews}

Thirty-six telephone or face-to-face semi-structured interviews were held with pre-hospital healthcare providers to explore their views on the essential components of an ALS course curriculum. The method of interview (ie. face-to-face or telephone) was selected on the basis of interviewee location and preference. Interviewees were identified as personal contacts of the researcher.

An interview protocol was developed to guide the interview including an introduction explaining the research, informed consent and interview questions. Interview participants were provided with a consent letter, which also outlined the key questions which were to be discussed in the interview. Interviews followed a semi-structured format allowing for particular participant views to be explored in-depth. The interview protocol was piloted with six paramedic academics prior to commencement. The duration of each interview was between 60 and 90 minutes. The interviews, held between June 2016 and October 2018, were semi-structured in nature and aligned to the consolidated criteria for reporting qualitative research (COREQ) (21). Contemporaneous written notes taken at the interviews, transcribed and entered into NVivo® for analysis. Interviews were coded, using a bracketing technique as suggested by Gearing (22) into themes based on words used by the interviewee (23).

The interviews used an interpretivist paradigm, which assumes that the views of all involved in the research was equally 'truthful' and based on the individuals' personal experiences of pre-hospital ALS (24). Having worked as a practitioner, the 
interviewers' experiences were grounded in patient care including performance of pre-hospital ALS and delivering ALS education to undergraduate students. The risk of bias or researcher assumptions was minimised by developing and using a standardised interview protocol from key themes identified in the literature review, bracketing interview results and aligning them to the literature, and including an expert panel which included non-paramedic experts in the analysis process.

\section{Expert panel}

An expert panel of 10 healthcare professionals were consulted face-to-face, via email or telephone, with discussions following the protocol of the previously described interviews. Given the expert nature of the panel, discussions allowed for individual panel members to provide detailed information in relation to pre-hospital ALS and the areas which were of most interest and considered important by them. The expert panel were recruited through face-to-face conversations and email following telephone calls, and chosen because of their knowledge of pre-hospital care, ALS and education. Each member of the expert panel was interviewed at least once, with two medical members interviewed twice, one nurse consulted four times, and the patient advocate twice. Informal conversations with the panel have also occurred as the research has progressed.

\section{Ethics approval}

This research was approved by Edith Cowan University's Human Research Ethics Committee, approval number 14928.

\section{Results}

Of the 38 respondents who completed the survey in full, $49 \%$ were operational paramedics and $41 \%$ were academics with pre-hospital experience. The remaining respondents were medical practitioners, nurses, or first responders with pre-hospital experience. Thirty-three face-to-face and three telephone interviews were also held with paramedics (educators and operational) (17 interviewees), registered nurses (two interviewees), medical practitioners (two interviewees), military personnel (one interviewee) and first responders (14 interviewees, including volunteer ambulance officers). Respondents were from Western Australia (21 interviews), the Northern Territory (nine interviews), New South Wales (two interviews), Northern Ireland (three interviews) and Victoria (one interview). Although a small sample was used, common themes were identified across the interviewees, indicating that data saturation was reached (25).

The expert panel of 10 healthcare professionals included five medical practitioners (three with experience in the prehospital field, one general practitioner and one anaesthetist), two registered nurses (both with education and pre-hospital experience), two paramedics (one operational and one in senior management) and one patient advocate. Towards the end of the analysis phase of this research one paramedic member withdrew from the project. Each member of the expert panel was interviewed at least once, with two medical members interviewed twice, one nurse consulted four times, and the patient advocate twice.

Using a weighted average score (WS) where a strongly disagree response was provided a weighting of 1 and strongly agree was provided a weighting of 5 . Figure 1 presents a summary of the respondents' views on what should be included in a pre-hospital course. Respondents identified that there should be pre-reading relating to the ARC ALS guidelines (WS 3.8) and a pre-course quiz on candidate knowledge of ALS guidelines (WS 3.8). There was variation in respondents'
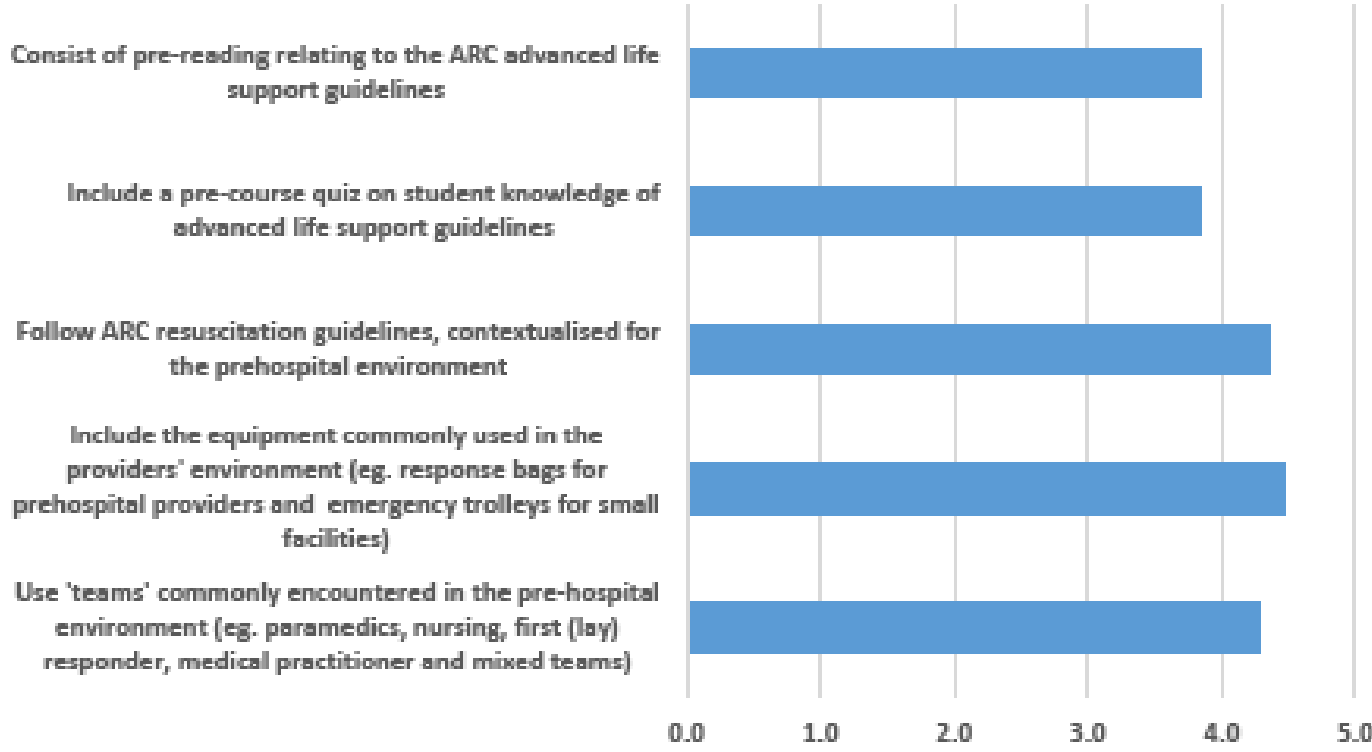

Figure 1. Weighted average score for course content 
views on the length of the quiz with respondents identifying that anywhere between 20 to 50 stem questions should be asked. The survey findings reflect the views of the interviewees and expert panel who indicated that the course should follow ARC guidelines and there should be a pre-course quiz of up to 40 questions.

Respondents to the survey, as well as interviewees and the expert panel indicated that a pre-hospital ALS course should use equipment commonly used in the providers' environment (WS 4.5). Respondents strongly agreed (WS 4.3) that teams should reflect those encountered in the pre-hospital environment:

'l'd gently suggest that the course could benefit patients by encouraging pre-hospital providers to view other healthcare providers and even bystanders as helpful partners under direction, given that cardiac arrest management is difficult, complex and has lots of things that need to be done simultaneously. I think there's a real opportunity for inter-professional understanding and cooperation.'

Survey respondents $(61 \%)$ identified that the length of a pre-hospital ALS course should be two days. However, interviewees and the expert panel identified that a pre-hospital ALS course should be one-day to teach core pre-hospital ALS principles and only be 2 days if specialist sessions involving a modified ALS approach, for example anaphylaxis, bariatric patients or paediatric cardiac arrest were to be included. The views of respondents can be summarised in the following interviewee response:

'An extra day could be added for those who are going to lead arrests or teach complex arrests (paediatric, bradycardias and trauma etc).'

Survey respondents agreed (WS 4.0) that a pre-hospital ALS course should be delivered in a mix of online and face-to-face modes. Half the expert panel indicated a pre-hospital ALS course should be taught entirely face-to-face, with the other half indicating the course should be taught in a mix of online and face-to-face modalities. In discussion with the expert panel, those indicating a mix of online and face-to-face were including pre-reading as an online component of the course, as shown in the following comment received:

'A mix would ensure that more time is spent on practical components in class/workshops.'

Respondents agreed that a pre-hospital ALS course should include theory lectures (WS 3.9) and case study discussions (WS 4.0). There was a strong preference from respondents for practical skills (WS 4.7) and practical scenarios (WS 4.7). The majority of respondents (WS 4.4) strongly agreed that a pre-hospital ALS course should follow ARC resuscitation guidelines contextualised for the pre-hospital environment. Interviewees and all but one of the expert panel indicated that a pre-hospital ALS course should be stand-alone covering those elements unique to the pre-hospital environment:

'Ensure that the course can be applied nationally. Keep the teaching broad and in line with ARC guidelines, and allow candidates to then apply the principles to their scope of practice.'

Of the theoretical elements of a pre-hospital resuscitation course, Figure 2 outlines those components which received the most support from survey respondents for inclusion in a pre-hospital ALS course. Human factors, an increasingly important aspect of healthcare delivery also were strongly supported as part of a pre-hospital ALS course are also outlined. The expert panel indicated that a pre-hospital ALS course should include elements of team communication and team leadership, however there was mixed support for critical thinking being included as a specific element of a pre-hospital ALS course. There was a view among the expert panel that healthcare professionals are already taught critical thinking skills, and as such this skill was already a part of the healthcare professional's standard approach to patient encounters.

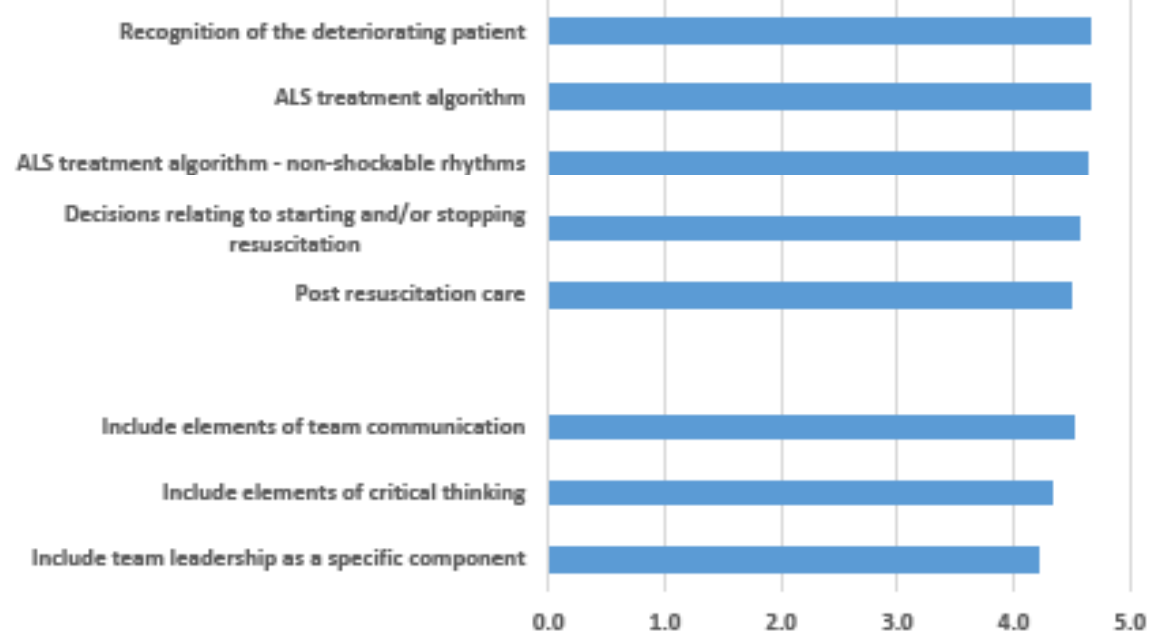

Figure 2. Theory elements and human factors (weighted score) 
Although there was general agreement from respondents that technical skills, such as drug administration, could be included in an ALS course, it was identified that these should be assumed knowledge. Interviewees and the expert panel clarified that skills sessions were not teaching candidates how to perform skills; rather the skills sessions were there to contextualise use of skills in the pre-hospital environment.

Scenarios were identified by survey respondents, interviewees and the expert panel as an important component of a prehospital ALS course. Over $90 \%$ of survey respondents as well as interviewees and the expert panel indicated shockable and non-shockable cardiac rhythms should be included. There was less support for inclusion of scenarios that required a modified or specialist ALS approach, with the exception of drowning. Interviewees identified that scenarios should focus on the reversible causes of cardiac arrest, teaching a standardised ALS approach which can be used in any pre-hospital situation. Outdoor locations ( $85 \%$ ), private areas such as homes or offices (79\%) and ambulance vehicles $(76 \%)$ were the three areas most supported by survey respondents, interviewees and the expert panel for location of pre-hospital ALS scenarios. The expert panel also identified small emergency department locations as being suitable for scenarios because of the resource limitations which occur in those environments. The majority of survey respondents (>70\%) agreed that scenarios should include up to four responders, including interprofessional healthcare teams and first responders, with less support for five or more responders. All interviewees and all but one of the expert panel also agreed that first responders or lay responders should be included in scenarios. The views of respondents can be summarised in the following feedback received:

'The pre-hospital setting is unique and differs from a hospital setting so should be delivered by subject matter experts in the field. The course should be generic to the pre-hospital environment without getting too specific on certain settings as this may become limiting.'

The majority of respondents (WS 4.4) and all interviewees and the expert panel agreed that assessment should occur on a continual basis through a pre-hospital ALS course. There was less support from respondents for single competence assessment at the end of the course. There was equal support for a theoretical assessment of knowledge either before or at the end of a pre-hospital ALS course as evidenced by the following comment:

'My understanding is that this is a practical skills course, so perhaps the theory should be assessed in a pre-course quiz as suggested. Continuous practical assessment in small chunks leading to an overall assessment at the end would work well.'

There was strong support from survey respondents that completion of a pre-hospital ALS course would result in an ARC statement of attainment (WS 4.4). Recertification was identified by survey respondents as having to occur every 2 years (40\%) or 3 years $(43 \%)$. Interviewees and the expert panel identified a 2-year recertification timeframe. Respondents to the survey indicated that a pre-hospital ALS course should be able to be taught by accredited universities (WS 4.1) or accredited ARC instructors (WS 3.7). These findings were confirmed by the expert panel as evidenced by the following comment:

'Whilst I'm not adverse to non-ARC instructors teaching on the course, there needs to be a governance process that operates at the individual level. There needs to be quality control of instructors and content and hence course outcomes, rather than a role recognition-based approach.'

\section{Discussion}

The aim of this research was to develop, based on past research, a survey and interviews, a curriculum for a prehospital ALS resuscitation course. Pre-reading is included in current ARC ALS courses. It would be expected that if a pre-hospital ALS course is following the current ARC approach, as suggested by this research, then pre-reading would also be included in a pre-hospital ALS course as a means to ensure that candidates have the necessary pre-requisite theoretical knowledge of relevant resuscitation guidelines. Current ARC courses also require a pre-course quiz to be undertaken as a means of establishing a baseline of candidates' theoretical knowledge. A pre-course quiz of up to 50 questions was suggested in the results of this research and is also identified as promoting congruency between current ARC courses and a pre-hospital ALS course.

The duration of the face-to-face component of current ARC courses is 1 (ALS1) and 2 (ALS2) days. Interviewees and the expert panel views indicated the length of a pre-hospital ALS course directly related to the volume of content to be included. This research suggests that the core of a pre-hospital ALS course should include non-shockable rhythms and shockable rhythms, and not scenarios requiring a modified ALS approach. These results suggest that a pre-hospital ALS course should be one-day in length.

Interviewees and the majority of the expert panel identified that a pre-hospital ALS course should be separate to a standard ALS course, covering those elements unique to the pre-hospital environment. Common across all survey respondents and interviewees was the view that a pre-hospital ALS course should be contextualised for the pre-hospital environment. This finding correlates with the literature, which suggests that education should reflect, as closely as possible, the environment in which course participants are going to be operating $(3,8)$. Following best practice as identified in the literature, the results from this research suggest that a pre-hospital ALS course should have a face-to-face element included $(1,6,7)$. A mixed approach would allow for pre-reading theory and lectures to be completed online, with the face-toface component then concentrating on skills and scenarios. 
The results of this research indicate that a pre-hospital ALS course should assume technical skill capability, and teach skill application in the pre-hospital environment. Variables such as cardiac arrest location and number of responders can be incorporated into each cardiac arrest response scenario as a means to embed the technical skills in a realistic environment. The simulation approach is well defined in the literature as a means of teaching without placing patients at risk (10-14).

The literature has identified that human factors are increasingly important component of healthcare $(9,15)$, and this research suggests that team communication and team leadership should be included in a pre-hospital ALS course. However, there was less agreement in relation to the inclusion of critical thinking, which interviewees in particular identified as already being taught as part of patient assessment and response.

The use of scenarios allow for theory, skills and human factors to be consolidated and practised in a physically and psychologically safe environment without risk to patients (1014). This research identified that a pre-hospital ALS course should include equipment commonly used in the pre-hospital environment and that teams should be the same as that encountered in the pre-hospital environment. Scenarios should focus on shockable rhythms and non-shockable rhythms. Specialist scenarios (such as pregnancy or trauma) could be left to a standard ALS course. Interviewees identified that teaching a standardised ALS approach which can be used in any pre-hospital situation would be beneficial. Outdoor locations, private areas such as homes, small emergency departments and ambulance vehicles were identified as being potential pre-hospital resuscitation simulation areas. Teams of different numbers and clinical skill, including the use of lay responders, was identified as being beneficial in a pre-hospital ALS course. The move towards interprofessional team working and communication enhancement has been highlighted in the literature $(9,16-19)$.

It is important that candidates complete assessment and receive feedback during educational courses $(5,9)$. It is harder to undertake formal assessment in short courses because short courses do not generally give candidates sufficient time to repeat and consolidate skills learned. This research suggests that competency should be assessed on a continual basis through a pre-hospital ALS course. It is important for professional development that candidates on continuing education courses obtain evidence of participation or competency for registration audit purposes. In this research there was strong support for the provision of a statement of attainment.

Education courses should be taught by professionals who are knowledgeable in the content and accreditation supports robust course governance and quality control (3-5). This research suggests that a pre-hospital ALS course could be taught by accredited ARC instructors or within accredited university degree courses. There are benefits and disadvantages to limiting who or where a pre-hospital ALS course could be taught, and if the aim is to increase consistency in pre-hospital ALS then making it available more widely to a suite of approved organisations may lead to positive patient outcomes and improved patient survival rates. The ARC recommends that basic life support be validated every 12 months with ARC ALS courses being valid for 3 years. The results of this research indicated that a pre-hospital ALS course should be revalidated every 2 or 3 years.

\section{Limitations}

The findings of this research, while valuable, must be considered in light of several limitations. First, the results are based on data collected from a small and non-randomly selected sample. The survey and semi-structured interviews focussed on perceptions and personal experiences of interviewees with respect to ALS. Different respondents may have alternate experiences and views. The primary researcher, who is an operational paramedic, has experience of pre-hospital ALS and may have unintentionally influenced the survey construct or interview analysis. The researcher was, however, aware of the potential for pre-existing bias to impact on study results and therefore based the survey on the previously-conducted literature review and employed bracketing techniques, as per the method suggested by Gearing (22) as part of the correlation between the literature, survey and interviews.

This research has only identified what could be included in a pre-hospital ALS course. This means the research does not yet show whether the suggested inclusions result in candidates who are better prepared to undertake pre-hospital ALS resuscitation. The final phase of this research will seek to examine whether the content of a pre-hospital ALS course results in candidates who are better prepared to undertake ALS in the pre-hospital environment.

\section{Conclusion}

The findings of this research suggest a pre-hospital ALS course should align with current ARC guidelines and include online precourse readings and a quiz. The course should a face-to-face element that should include lectures, case studies, skills and scenarios based on pre-hospital ALS situations. A pre-hospital ALS course should be generic enough so as to teach the principles of ALS, including human factors, in the pre-hospital environment which can then be transferred to the participants' work environment.

A pre-hospital ALS course should focus on core ALS skills including shockable and non-shockable rhythms. Resuscitation skills should be assumed knowledge, however contextualised for the pre-hospital environment. Scenarios should be 
undertaken in a range of locations including indoor and outdoor locations. Varying numbers of responders with different clinical skills, including lay responders, should be included in the scenarios. Assessment should consist of the pre-course quiz and continuous competency assessment during the course itself. A pre-hospital ALS course should also be able to be taught by accredited ARC instructors and in accredited undergraduate paramedicine degree courses, with participants receiving at least a statement of attendance, requiring revalidation every 2 to 3 years.

\section{Acknowledgements}

The authors wish to thank all the providers who responded to the survey and the members of the expert panel who took extensive time to speak with the researcher.

\section{Competing interests}

Professor Grantham and Mr Gale are Council members of the Australian Resuscitation Council. Professor Sim, Associate Professor Beatty and Mr Reid have no competing interests.

\section{References}

1. Reid D, Jone R, Sim M. Pre-hospital advanced life support education - core components for pre-hospital professionals. Australasian Journal of Paramedicine 2018;15(1).

2. Lyon RM, Sinclair N. National standards in prehospital resuscitation training are required. Emerg Med $\mathrm{J}$ 2012;29:602.

3. Australian Resuscitation Council (NSW Branch). (Undated). Cardiac arrest - an introduction. Sydney. Available at: http:// arcnsw.org.au/files/4913/5088/1980/Cardiac_Arrest_-_intro_ summary_-_ARC_NSW_document.pdf

4. Perkins G, Fullerton JN, Davis-Gomez N, et al. The effect of pre-course e-learning prior to advanced life support training: a randomised controlled trial. Resuscitation 2010;81:877-81.

5. Williams NM. Advanced life support training and assessment: a literature review. Australas Emerg Nurs J 2011;14:240-5.

6. Bonnetain E, Boucheix J, Hamet M, Freysz M. Benefits of computer screen-based simulation in learning cardiac arrest procedures. Med Educ 2010;44:716-22.

7. Christenson J, Parrish K, Barabé S, et al. A comparison of multimedia and standard advanced cardiac life support learning. Acad Emerg Med 1998;5:702-8.

8. Gale M, Grantham H, Morley P, Parr M. Advanced life support level 2. Perth: Australian Resuscitation Council; 2016.

9. Gjerra K, Moller TP, Ostergaard D. Efficacy of simulation- based trauma team training of non-technical skills. A systematic review. Acta Anaesthesiol Scand 2014;58:77587.

10.Bredmose PP, Habig K, Davies G, Grier G, Lockey DJ. Scenario based outdoor simulation in pre-hospital trauma care using a simple mannequin model. Scand J Trauma Resusc Emerg Med 2010;18:13.

11.Lumsden H. Newborn life support course: training students. Br J Midwifery 2008;16:717-21.

12. Hoadley TA. Learning advanced cardiac life support: a comparison study of the effects of low- and high-fidelity simulation. Nurs Educ Perspect 2009;30:91-5.

13.Aqel AA, Ahmad MM. High-fidelity simulation effects on cpr knowledge, skills, acquisition, and retention in nursing students. Worldviews Evid Based Nurs 2014;11:394-400.

14.Davis LE, Storjohann TD, Spiegel JJ, Beiber KM, Barletta JF. High-fidelity simulation for advanced cardiac life support training. Am J Pharm Educ 2013;77:59.

15. Von Wyl T, Zuercher M, Amsler F, Walter B, Ummenhofer W. Technical and non-technical skills can be reliably assessed during paramedic simulation training. Acta Anaesthesiol Scand 2009;53:121-7.

16. Husebø SE, Rystedt H, Friberg F. Educating for teamwork - nursing students' coordination in simulated cardiac arrest situations. J Adv Nurs 2011;67:2239-55.

17.Dagnone JD, McGraw RC, Pulling CA, Patteson AK. Interprofessional resuscitation rounds: a teamwork approach to ACLS education. Med Teach 2008;30:e49-54.

18. Birkhoff SD, Donner C. Enhancing pediatric clinical competency with high-fidelity simulation. J Contin Educ Nurs 2010;41:418-23.

19. Garbee DD, Paige J, Barrier K, et al. Interprofessional teamwork among students in simulated codes: a quasiexperimental study. Nurs Educ Perspect 2013;34:339-44.

20. Eysenbach $\mathrm{G}$. Improving the quality of web surveys: the checklist for reporting results of internet e-surveys (CHERRIES). J Med Internet Res 2004;6(3).

21. Tong A, Sainsbury P, Craig J. Consolidated criteria for reporting qualitative research (COREQ): a 32 item checklist for intrdviews and focus groups. Int J Qual Health Care 2007; 19:349-57.

22.Gearing R. Bracketing in research: a typology. Qual Health Res 2004;14:1429-52.

23. Neutens J. Research techniques for the health sciences. 5th edn. Sydney: Pearson; 2014.

24.Cohen D, Crabtree B. Qualitative research guidelines project New Jersey: Robert Wood Johnson Foundation; 2006. Available at: www.qualres.org/Homelnte-3516.html

25. Saunders B, Sim J, Kingstone T, et al. Saturation in qualitative research: exploring its conceptualization and operationalization. Qual Quanti 2018;52:1893-907. 